\section{Giant chondroma of the sternum mimicking a mediastinal mass}

\author{
Gershon Fink, Michael Bergman, \\ Moris Levy, Irena Avidor, Shimon Spitzer
}

\begin{abstract}
A 64 year old man with a giant benign sternal chondroma presented with cough as his sole complaint.
\end{abstract}

Primary benign sternal tumours are uncommon. ${ }^{12}$ O'Neal and Ackerman ${ }^{3}$ reviewed 85 cartilaginous lesions of ribs and sternum that had been reported up to 1950 and added 11 of their own. Eight of the 96 cases were reported to be benign tumours of the sternum, though the benignity was questioned in four. Of 2000 primary bone tumours reviewed by Pascuzzi et $a l^{4}$ in 1957 in the Mayo clinics, only one sternal tumour was classified as benign. In a review of 134 cases of tumours of the thoracic skeleton in 1966 only one lesion of the sternum was benign (eosinophilic granuloma). ${ }^{5}$ Sabanathan et al found two benign sternal chondromas among 53 primary chest wall tumours. ${ }^{6}$ Additional isolated cases of benign sternal tumours have been reported. ${ }^{78}$ In reviews before 1940 many benign cartilaginous neoplasms of ribs and sternum with repeated recurrences were described. Of 36 patients listed as having a benign tumour before 1950 , only three were alive and well five years after surgery. ${ }^{4}$ We report a case of giant benign sternal chondroma.

\section{Case report}

A 64 year old non-smoking carpenter complained of a non-productive cough and dyspnoea on exertion of two months duration. He had a history of hypertension, treated by amiloride hydrochloride and hydrochlorthiazide, and type II diabetes mellitus, treated by glibenclamid. On physical examination the patient appeared well and the only abnormality was distended jugular veins. The lungs were clear.

A chest radiograph showed a large mediastinal shadow bulging on both sides of the mediastinum, more towards the left side (fig 1a). Computed tomography of the chest showed a large retrosternal mass in the anterior and middle mediastinum (fig $1 b$ ). Pulmonary function tests showed a restrictive ventilatory impairment (vital capacity $67 \%$ and total lung capacity $72 \%$ of predicted values). The maximal flow rates were normal. There was mild hypoxaemia (arterial oxygen tension $9.0 \mathrm{kPa}$ ) and normal carbon monoxide transfer factor.

A median sternotomy disclosed a large

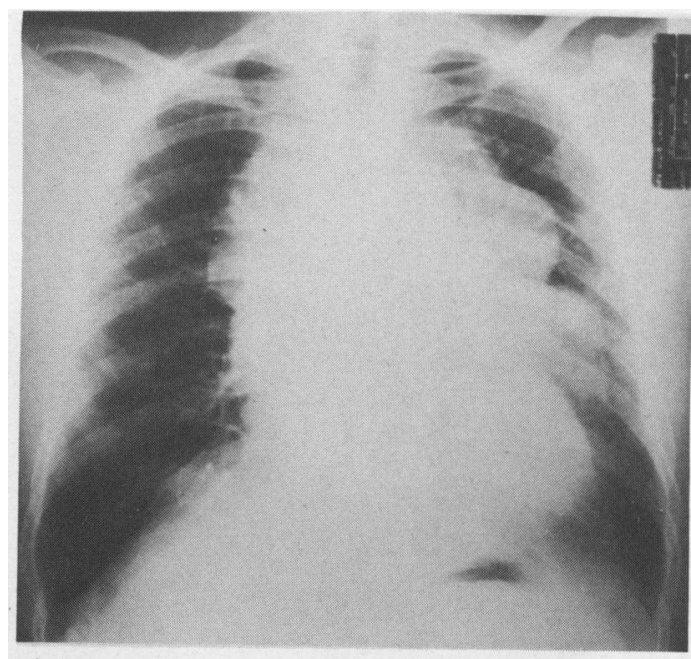

(a)

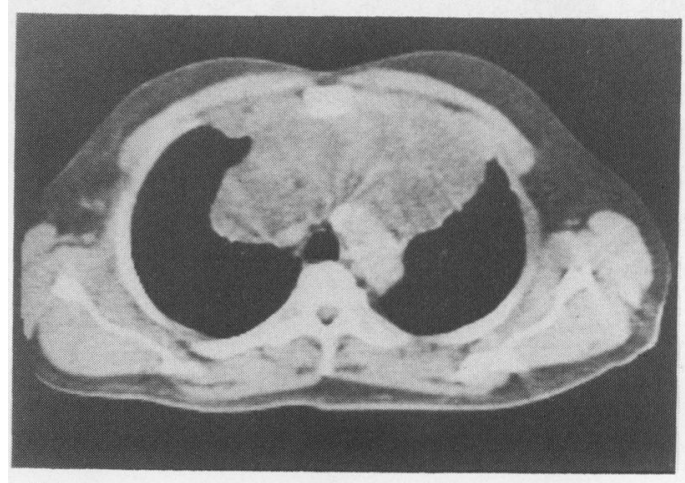

(b)

Figure 1 (a) Chest radiograph and (b) computed tomogram of the chest before surgery.

growth, $16 \times 14 \times 13 \mathrm{~cm}$ in size, consisting of about 20 cystic masses in the mediastinum, overlying the ascending aorta, the aortic arch, the pericardium, the superior vena cava, and the pleural cavities. The masses arose from the internal wall of the sternum and included solid and cystic masses containing yellow mucoid material. Radical excision of the growth was performed.

Histological sections confirmed the diagnosis of chondroma, with no evidence of malignancy (fig 2).

The patient recovered and shows no evidence of recurrence four years after surgery.

\section{Discussion}

Benign chondroma of the sternum is a very rare lesion with less than a dozen cases reported in Western publications. The presenting symptoms in previous reports have been chest pain and external deformation of the sternum. ${ }^{367}$ Most of these tumours have been relatively small, less than $10 \mathrm{~cm}$ in diameter. ${ }^{37}$

Our patient differs in that his presenting symptom was cough. There was no local deformity of the sternum to suggest the origin of the tumour. The appearance was very unusual because of the huge size of the chondroma, which arose from the internal surface of the sternum and mimicked a mediastinal polycystic mass. The restrictive ventilatory defect was presumably due to the large size of the tumour. 


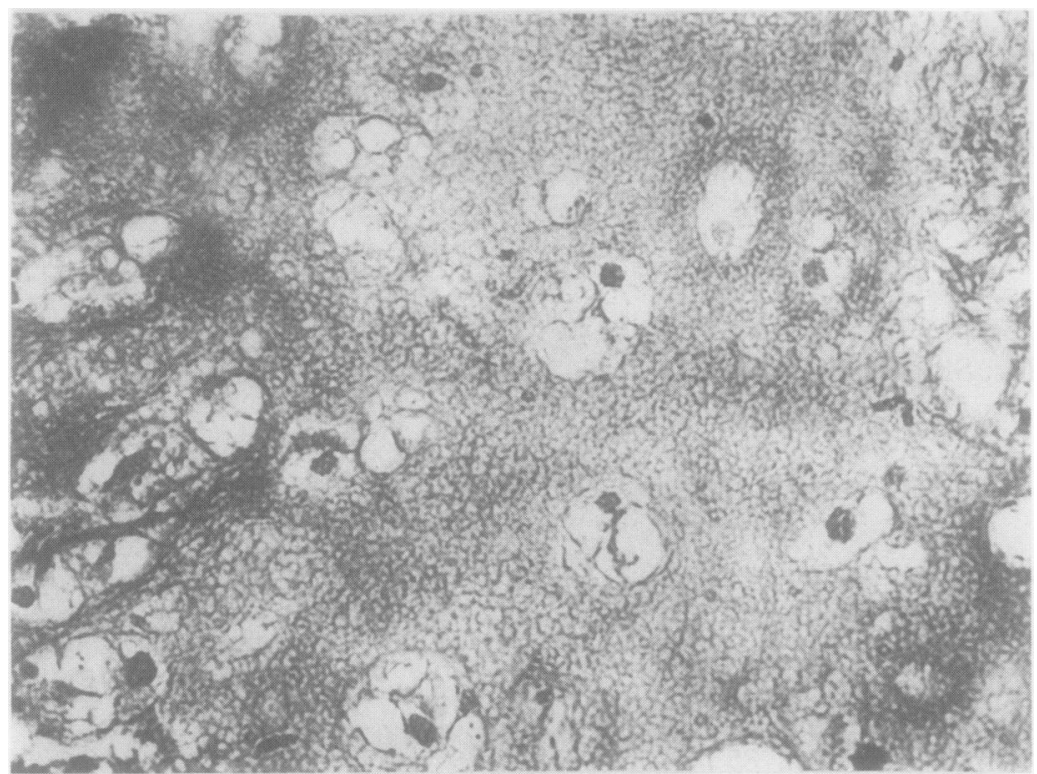

Figure 2 Photomicrograph showing groups of cartilage cells. (Haematoxylin and eosin.)
The mass was radically extirpated and the lack of recurrence in the four years since surgery is consistent with the pathological diagnosis of benignity.

1 Stelzer P, Gay WA.Tumors of the chest wall. Surg Clin North Am 1980;60:779-91.

2 Vieta JO, Maier HG. Tumors of the sternum. Surg Gynecol Obstet 1962;114:513-25.

3 O'Neal LW, Ackerman LV. Cartilaginous tumors of ribs and sternum. $J$ Thorac Surg 1951;21:71-108.

4 Pascuzzi CA, Dahlin DC, Clagett OT. Primary tumors of the ribs and sternum. Surg Gynec Obstet 1957;104: $390-400$

5 Ochsner A Jr, Lucac GL, McFarland GB. Tumors of the thoracic skeleton: review of 134 cases. J Thorac Cardiovasc Surg 1966;52:311-21.

6 Sabanatham S, Salama FD, Morgan WE, Marvey JA. Primary chest wall tumors. Ann Thorac Surg 1985;39: 4-15.

7 Katz S. Sternal chondroma. Dis Chest 1966;55:166-9.

8 Alonso-Sej F, De Linera FA. Resection of the entire sternum and replacement with acrylic resin. Report of a case of giant chondromyxoid fibroma. J Thorac Cardiovasc Surg 1971;62:271-9. 